\title{
Analysis of Photoluminescence measurement data from interdiffused Quantum Wells using Adaptive Real coded Quantum inspired Evolutionary Algorithm
}

\author{
Ashish Mani ${ }^{1}$ \\ Sr. Lecturer, USIC \\ Dayalbagh Educational Institute, Dayalbagh, Agra - 282110, UP, India \\ E-mail:mani.ashish@gmail.com \\ C. Patvardhan \\ Professor, Department of Electrical Engineering \\ Dayalbagh Educational Institute, Dayalbagh, Agra - 282110, UP, India \\ E-mail: cpatvardhan@gmail.com
}

\begin{abstract}
:
Reliable analysis of any experimental data is always difficult due to the presence of noise and other types of errors. This paper analyzes data obtained from photoluminescence measurement, after annealing of interdiffused Quantum Well hetrostructures, using an Adaptive Real coded Quantum inspired Evolutionary Algorithm (ARQiEA). The proposed algorithm directly computes interdiffusion parameters without using Arrhenius plot. Further, the results obtained have been compared with those reported with Genetic Algorithm and Least Square Analysis methods. The ARQiEA is suitable for parameter estimation problems as it is free from parameter fine-tuning and provides result with least overall error.
\end{abstract}

13th International Workshop on Advanced Computing and Analysis Techniques in Physics Research - ACAT 2010 Jaipur, India

February 22-27 2010

\footnotetext{
$1 \quad$ Speaker
} 


\section{Introduction}

Measurement process is often fraught with various types of errors and presence of noise due to which reliable analysis of experimental data is an important and challenging task. This paper analyzes data obtained from photoluminescence measurements, after annealing of interdiffused Quantum Well hetrostructures, using Adaptive Real coded Quantum inspired Evolutionary Algorithm (ARQiEA).

Photoluminescence (PL) is a widely used process for measurement of interdiffusion parameters in semiconductor quantum well hetrostructures [1]. This method correlates the changes of the confined energy levels (PL peak energy) into characteristic diffusion length $\left(L_{D}\right)$ of the quantum well structure by a linear theoretical model. The validity of this model is ascertained by plotting the square of the correlated diffusion length against annealing time. Subsequently, least square analysis (LSA) fit is mostly required to extract the interdiffusion coefficients at given annealing temperatures due to presence of noise and errors in experimental data. Further, Arrhenius plot is used for determining the activation energy (Ea) and prefactor (Do) of the interdiffusion process from the interdiffusion coefficients at given anneal temperatures by applying LSA again [2]. The application of this method often results in determination of interdiffusion parameters that are not in consonance with the theoretical model as the straight line fits to square of the correlated diffusion length against annealing time exhibits nonzero intercepts [2], [3], and [4].

Genetic Algorithm was proposed in [5] as an alternative for LSA fit to overcome the above-mentioned problem due to experimental errors. It also simplified the determination of interdiffusion parameters as a single step process without using Arrhenius plot. However, this implementation of GA employed both crossover and mutation operators, which had user selectable parameters. This paper proposes the use of an Adaptive Real coded Quantum inspired Evolutionary Algorithm (ARQiEA), which is free from user selectable parameters as these are adaptively determined during the search process. The proposed algorithm is more suitable for data analysis purpose especially for the non-specialist groups, as it does not require domain information either in Evolutionary algorithms (EA) or in application area to set the EA parameters.

The paper is organized as follows. Section 2 introduces Evolutionary Algorithms. QiEA are introduced in Section 3. Section 4 describes the proposed ARQiEA. Section 5 discusses the problem formulation for PL measurement data analysis. Testing methodology and Results are discussed in Section 6. Section 7 summarizes the findings of this effort.

\section{Evolutionary Algorithms}

Evolutionary Algorithms are a collection of stochastic unsupervised problem-solving paradigms, guided by the natural process of evolution [6].They have been successful in application areas where traditional deterministic techniques are rendered useless due to lack of proper domain information and near optimal solutions are acceptable [7]. EA is a populationbased technique, which simulates the evolution process via operators such as selection, 
recombination and mutation. A population of candidate solutions competes for survival from one iteration to the next with new candidate solutions being generated at each iteration, the population gradually moves to the optimal or near-optimal solution in the search space. Individual members of the population representing candidate solutions are typically strings of binary or real-valued parameters, although other representations such as trees, linked lists, etc. can also be created [8].

The pseudo code for canonical EA is given in figure 1.

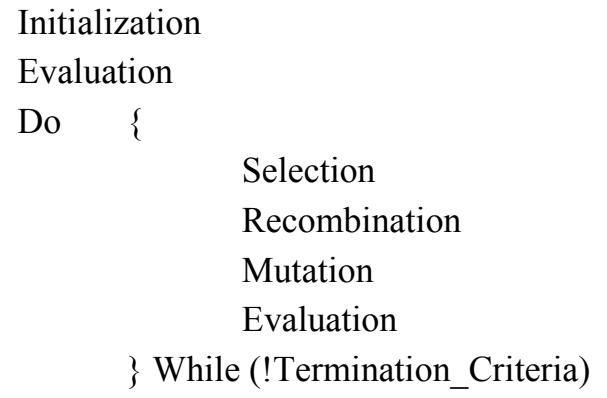

Figure 1: Pseudo Code for Canonical Evolutionary Algorithm

Selection focuses on healthy individuals by exploiting the available fitness information. Recombination and mutation utilizes the current individuals to evolve new solutions. Recombination selects individuals on the basis of their relative fitness such that fitter individuals have a higher probability of reproducing than unsuccessful ones. Further, it may combine two or more individuals to produce new potential solutions. Mutation perturbs the individuals to maintain the diversity of the population, and is also responsible for exploration. Local search heuristics may also be used for improving the convergence speed.

EAs are different from traditional search and optimization paradigms as they employ a population of potential solutions, have black box nature and decisions are probabilistic rather than deterministic. These are black box techniques and therefore, guided by the fitness of the individuals only [7].They are popular due to their simplicity and ease of implementation. However, they suffer from problems like premature convergence, slow convergence, stagnation and are sensitive to the choice of the crossover and mutation operators and parameters.

Convergence in EAs can be explained as a phenomenon in search process when all the individuals are localized in a small region of search space and no further improvement can be achieved by employing recombination operators alone. Premature convergence implies that all the individuals in the population have converged to a suboptimal solution. Slow convergence implies that search process converges to the optimal but very slowly i.e. consuming too much time and resources. Stagnation means that search process tends to remain stuck in a particular local optimum for long duration i.e. no observable improvement is made in solution quality for long durations during the search process. However, this does not imply that search process has lost the power to reach global optimum, as is the case in premature convergence.

Parameters selection includes finding values of crossover rate, mutation rate and operator associated parameters etc. to design an EA for solving specific problems. The values of these 
parameters ascertain the efficacy of the search process. Parameter tuning and control is a challenging task as this problem is not only important for success of the EA but at the same time is poorly structured, ill defined and complex [9].

A lot of effort has been made by the researchers to overcome the convergence issues by establishing a better balance between Exploitation and Exploration. Quantum inspired Evolutionary Algorithm (QiEA) [10] has also been proposed to improve the balance between exploration and exploitation.

The problems associated with parameter selection are mostly solved by experimental trial and error methods. Feedback from search process has been used for adaptively controlling some parameters [9]. An Adaptive Real Coded Quantum inspired evolutionary algorithm [11] described in the next section is proposed to solve some of the problems associated with parameter selection.

\section{Quantum Inspired Evolutionary Algorithms}

The quantum-computing paradigm has been shown to be more powerful than classical computing. It has also been used for improving classical stochastic algorithms. Quantum inspired Evolutionary algorithms (QiEA) are such an effort at integrating the principles of quantum computing and Evolutionary Algorithms to solve some problems associated with canonical EAs.

QiEAs are evolutionary algorithms developed by drawing some ideas from the principles of Quantum Mechanics and hybridizing them in the current framework of EAs. Thus, QiEAs are not pure quantum algorithms but a new type of EA that can be run on classical computers. Thus, QiEAs do not require yet to be commercially available Quantum Computers for their implementation and execution.

Some important principles of Quantum mechanics are superposition, entanglement, interference and measurement [12]. These principles have been mostly used in inspiring the representation of the solution, crossover and mutation operators [13]. The principles, which have been mostly utilized in designing QiEAs, are superposition and measurement [10]. These have been primarily used for quantum representation of search strings for improving diversity as reported in literature.

Narayanan and Moore proposed quantum-inspired genetic algorithm by integrating Quantum Parallel verses Interference with genetic crossover operator to solve Travelling Salesman problem [14]. Han and Kim introduced qubit representation of solution and also used inspiration from quantum measurement principle and phase rotation quantum gates to develop QiEA for solving combinatorial optimization problems [10]. They further improved the phase rotation quantum gate and introduced a new termination criterion [15]. It was claimed that QiEA maintained better balance between Exploration and Exploitation due to the probabilistic nature of the qubit. However, these attempts used binary string representation and designer's specified parameters for which choice is governed by the nature of the problem. Platlet et al. have shown the weaknesses of Han and Kim's QiEA and proposed a new algorithm using the same probabilistic representation, called versatile quantum- inspired evolutionary algorithm (vQEA) [16]. They claim that the new algorithm always adapts search towards the last 
promising solution found thus leading to a smoother and more efficient exploration. This claim is supported by experimental evaluations.

An interesting point of such implementation is no direct correspondence between the solution vector and qubits especially in the case of real coded QiEA [17], [10]. The quantum rotation gates / operators also behave independent by the information from the problem and solution domain assuming that the quantum behavior would help in reaching the solution. However, it should not be forgotten that such algorithms are to be run on classical computers without simulating any quantum phenomena. Further, it can be argued that increasing the diversity by collapsing the solution qubit may affect the exploitation of the solution as the solution found in the next iteration even of a good candidate solution may end up being far worse due to the probabilistic implementation. Some of these problems are remedied in ARQiEA presented in the next section.

\section{Adaptive Real coded Quantum inspired Evolutionary Algorithm}

This paper takes a different approach for designing the QiEA by using not only qubit representation and the associated superposition principle but also the entanglement principle. Entanglement is one of the fundamental principles of Quantum Computing. If two qubits are entangled then performing any quantum operation on one of the qubits will affect the state of the other qubit as well i.e. there is a relation between the two qubits, which can be utilized for computation purposes. The proposed ARQiEA uses two qubits per solution vector to utilize entanglement principle. The entanglement principle is being integrated in a classical algorithm, so the implementation is classical.

The proposed ARQiEA tries to overcome the limitations typically associated with EA implementations. EAs have only an objective function value as domain information regarding a specific problem. This feedback is mostly used in the selection phase and not for directly controlling the crossover or mutation operators or even local heuristic. Therefore, the feedback through objective function value is not being utilized completely.

The proposed algorithm uses the second qubit to store the information regarding the objective function value of the solution vector stored in first qubit. This provides information regarding the solution domain as well as the objective function domain made available simultaneously. The information stored in the first and the second qubit is in entanglement and thus harness the power of the important entanglement principle. The first qubit influences the second qubit as the first qubit determines the objective function value and hence the second qubit. The second qubit influences the first qubit as the parameter free adaptive quantum rotation crossover operator used for evolving the first qubit uses the second qubit to determine the degree of rotation. Any operation performed on either of the two qubits would affect the other and so they are in entanglement, but implementation is classical.

\section{Representation}

The smallest information element in quantum computer is a qubit, which is quantum analog of classical bit. The classical bit can be either in state 'zero' or in state 'one' whereas a 
quantum bit can be in a superposition of basis states in a quantum system. It is represented by a vector in Hilbert space with $\mid 0>$ and $\mid 1>$ as the basis states. The qubit can be represented by vector $|\psi\rangle$, which is given by:

$$
|\psi>=\alpha| 0>+\beta \mid 1>
$$

where $|\alpha|^{2}$ and $|\beta|^{2}$ are the probability amplitude of qubit to be in state $\mid 0>$ and $\mid 1>$ respectively and should satisfy the condition:

$$
|\alpha|^{2}+|\beta|^{2}=1
$$

The qubits can store, in principal exponentially more information than classical bits. However, these qubits exist in quantum computing systems and are constrained by several limitations such that they collapse to one of the basis states upon measurement and can be evolved by using unitary transformations. The simulation of qubits is inefficient on classical computers.

In QiEA, the probabilistic nature of qubits has been widely used for maintaining diversity [10]. A single qubit is attached to the solution vector and the solution is obtained by taking measurement or collapsing in binary coded as well as real coded QiEA [17]. However, due to reasons explained in the previous section, this paper uses a real coded representation of the solution with two sets of qubits. The first set of qubits $\left|\psi_{1 \mathrm{i}}\right\rangle$ stores the current value of the $\mathrm{i}^{\text {th }}$ variable as amplitude $\alpha_{1 i}$ whose value is $[0,1]$. The upper and lower limits of the variables are scaled between zero and one. The amplitude $\beta$ is not stored as it can be computed from equation 2 . Therefore, the number of qubits per quantum register $\mathrm{QR}_{1}$ is equal to the number of variables. The quantum register stores the qubits. The number of quantum registers is also a function of the number of variables in the specific problem creating a problem bias rather than a user bias. The number of $\mathrm{QR}_{1}$ is 5 times the number of variables. The number of $\mathrm{QR}_{1}$ is kept high in order to utilize the benefit of superposition and quantum rotation in EA. The structure of $\mathrm{QR}_{1}$ is shown below:

$$
\begin{aligned}
& \mathrm{QR}_{11} \quad=\quad\left[\alpha_{111}, \alpha_{112} \ldots \alpha_{11 \mathrm{n}}\right] \\
& \mathrm{QR}_{15 \mathrm{~N}}=\left[\alpha_{15 \mathrm{n} 1}, \alpha_{15 \mathrm{n} 2} \ldots \alpha_{15 \mathrm{nn}}\right]
\end{aligned}
$$

The second set of Qubits $\mid \psi_{2 i}>$ stores the ranked and scaled objective function value of the ith solution vector as amplitude $\alpha_{2 \mathrm{i}}$ whose value is $[0,1]$. The fittest vector's objective function value is assigned the value one and that of the worst vector is assigned the value zero. The rest of the solution vectors' objective function values are ranked and assigned relative values between zero and one.

\section{Entanglement and Superposition}

Quantum Entanglement is a property of a quantum mechanical system with two or more qubits in which the quantum states of one qubit influences the other even if they are spatially 
separated [12]. It is widely believed that Entanglement is one of the reasons of Quantum Computers being more powerful than their classical counterparts. The mathematical representation of the classical implementation of superposition and entanglement principle is:

$$
\begin{aligned}
& \left|\psi_{2 \mathrm{i}}(\mathrm{t})\right\rangle=\mathrm{f}_{1}\left(\mid \psi_{1 \mathrm{i}}(\mathrm{t})>\right) \\
& \left|\psi_{1 \mathrm{i}}(\mathrm{t}+1)>=\mathrm{f}_{2}\left(\left|\psi_{2 \mathrm{i}}(\mathrm{t})\right\rangle,\left|\psi_{1 \mathrm{i}}(\mathrm{t})>,\right| \psi_{1 \mathrm{j}}(\mathrm{t})>\right)\right.
\end{aligned}
$$

where $\left|\psi_{1 \mathrm{i}}\right\rangle$ and $\left.\psi_{1 \mathrm{j}}\right\rangle$ are the first qubits associated with the $\mathrm{i}^{\text {th }}$ and $\mathrm{j}^{\text {th }}$ solution vectors respectively, $\left|\psi_{2 \mathrm{i}}\right\rangle$ is the second qubit associated with the $\mathrm{i}^{\text {th }}$ solution vector, $\mathrm{t}$ is iteration number, $f_{1}$ and $f_{2}$ are the functions through which both the qubits are classically entangled. The superposition principle is harnessed classically by ranking and scaling the second qubit such that the second qubit is in superposition of the solution vector's i.e. first qubit. Similarly, the first qubit is also scaled uniformly between zero and one.

\section{Adaptive Quantum inspired Crossover Operator}

Quantum gates are used for evolving the qubits in quantum computing paradigm. Quantum phase rotation gate is used in Grover's algorithm for amplitude amplification for searching the marked element in the unsorted database [12]. Most of the efforts in QiEAs have used rotation gates for evolving the qubits. These operator's parameters are mostly dependent on the problem. Further, the past efforts have also used mutation operator and local heuristics [13].

A quantum rotation based adaptive and parameter tuning free crossover operator has been designed by hybridizing quantum entanglement with modified BLX- $\alpha$ crossover operator [18]. The second qubits' amplitude is used for determining the angle of rotation for evolving the first qubit. The following equation is used for the purpose:

$$
\psi_{1 i}(t+1)=\psi_{1 i}(t)+f\left(\psi_{2 i}(t), \psi_{2 j}(t)\right) *\left(\psi_{1 j}(t)-\psi_{1 i}(t)\right)
$$

where $t$ is the iteration number, $\psi_{1 \mathrm{j}}$ can be the best solution vector or any other randomly or deterministically selected solution vector depending on the specific rotation strategy. All solution vectors are rotated towards best solution vector when $\psi_{1 \mathrm{j}}$ is the best solution. When the solution vector is randomly or deterministically picked, the inferior solution is rotated towards the better solution. In case of the best solution, it is rotated away from the inferior solution. Thereby, the quantum inspired crossover operator balances the exploration and exploitation and converges the solution vector adaptively towards global optima by using three strategies:

Rotation towards Best Strategy (R-I) is implemented by selecting each solution vector (individual) sequentially except for the best solution vector which is used as the pivot towards which all the other solution vectors are rotated. The main motivation behind this strategy is the conjecture that there is a high probability of improving all the other solution vectors by searching towards the best vector. Thus, in the initial stages of the search, this strategy helps in exploration. However during the later stages, when the 
solution vectors are now mostly located in a smaller region, this strategy helps in exploitation.

Rotation away from Worse Strategy (R-II) is implemented by selecting each solution vector (individual) sequentially except for the best solution vector, which is rotated away from all the other solution vectors. This strategy is primarily used for the exploitation purpose, as there is a high probability of improving the best solution vector by searching in its vicinity. The search takes place simultaneously in all the dimensions due to the population of all the other solution vectors.

Rotation Towards Better Strategy (R-III) is implemented by selecting any two solution vectors randomly and rotating the inferior one towards the better solution vector. This strategy is primarily used for the exploration purpose, as there is uniformly guided search in the entire solution space for reaching the regions closer to the optimum. It can be appreciated that, this is not a blind search as is the case with the mutation operator in canonical EAs.

The function $\left.\mathrm{f}\left(\psi_{2 \mathrm{i}}(\mathrm{t}), \psi_{2 \mathrm{j}} \mathrm{(t}\right)\right)$ in equation 5 controls gross and fine search. Presently, $\mathrm{f}\left(\psi_{2 \mathrm{i}}(\mathrm{t})\right.$, $\left.\psi_{2 \mathrm{j}}(\mathrm{t})\right)$ generates a random number either between 0 and $\alpha_{2 \mathrm{i}}-\alpha_{2 \mathrm{j}}$ or between 0 and $\left|\alpha_{2 \mathrm{j}}\right|^{2}-\left|\alpha_{2 \mathrm{i}}\right|^{2}$. The value $\left|\alpha_{2 j}\right|^{2}-\left|\alpha_{2 i}\right|^{2}$ is generally smaller than $\alpha_{2 \mathrm{j}}-\alpha_{2 \mathrm{i}}$, thus the latter is used for gross search and former for fine search. The salient feature of the new quantum inspired crossover operator is that it adaptively changes each variable in the solution vector and at the same time is problem driven rather than being an arbitrary choice of the user.

\section{Pseudo code for Proposed ARQiEA}

1. Initialize Quantum inspired Register $\mathrm{QR}_{1}$.

While (!termination_criteria) \{

2. Compute fitness of $\mathrm{QR}_{1}$.

3. Assign $\mathrm{QR}_{2}$ on the basis of the fitness of $\mathrm{QR}_{1}$.

4. Perform Adaptive Quantum inspired Crossover on $\mathrm{QR}_{1}$.

5. Select next generation $\mathrm{QR}_{1}$ using elitist strategy .

\section{Description}

1. Initialize the first set of qubits in the quantum register $\mathrm{QR}_{1}$ randomly. $\mathrm{QR}_{1}$ stores $\alpha$ 's corresponding to the solution vectors scaled between $[0,1]$ of the population.

2. Compute the fitness of each solution vector of $\mathrm{QR}_{1}$ by using the equation 9 in section 4 .

3. $\mathrm{QR}_{2}$ stores the ranked and scaled objective function value of the ith solution vector in $\mathrm{QR}_{1}$ as amplitude $\alpha_{2 \mathrm{i}}$ whose value is in the range $[0,1]$. The fittest vector's objective function value is one and of the worst vector is zero. The rest of the solution vectors' objective function values are ranked and assigned values between the zero and one.

4. Adaptive Quantum Crossover is performed by using all the three strategies R-I, R-II and $\mathrm{R}-\mathrm{III}$ on $\mathrm{QR}_{1}$. 
5. The solution vector for the next generation are selected by comparing individual parents with their best child and applying tournament selection i.e. the fitter one makes it to the next generation.

6. Termination criterion is based on the maximum number of iterations, which in this implementation is 1000 .

\section{Problem Formulation}

Photoluminescence (PL) measurement process correlates the changes of the confined energy levels (PL peak energy) into characteristic diffusion length $\left(\mathrm{L}_{\mathrm{D}}\right)$ of the quantum well structure by a linear theoretical model. The correlated $\mathrm{L}_{\mathrm{D}}^{2}$ is plotted against annealing time, $\mathrm{t}$, to determine the interdiffusion coefficient, D (T), by using the following equation: [5]

$$
\mathrm{L}_{\mathrm{D}}^{2}=4 * \mathrm{D}(\mathrm{T}) * \mathrm{t}
$$

The interdiffusion parameters viz., activation energy, $\mathrm{E}_{\mathrm{a}}$, and the interdiffusion prefactor, $\mathrm{D}_{\mathrm{o}}$, are determined by using Arrhenius equation:

$$
\mathrm{D}(\mathrm{T})=\mathrm{D}_{\mathrm{o}} * \exp \left(-\mathrm{E}_{\mathrm{a}} /\left(\mathrm{K}^{*} \mathrm{~T}\right)\right)
$$

where $\mathrm{K}$ is Boltzmann Constant and $\mathrm{T}$ is the annealing temperature in Kelvin.

By combining equations(6) and (7), the following equation is obtained:

$$
\mathrm{L}_{\mathrm{D}}^{2}(\mathrm{t}, \mathrm{T})=4 * \mathrm{t} * \mathrm{D}_{\mathrm{o}} * \exp \left(-\mathrm{E}_{\mathrm{a}} /(\mathrm{K} * \mathrm{~T})\right)
$$

Thus, the fitness function required for the proposed algorithm is squared error as given in [5] for collective runs i.e. taking the measurement data at different annealing temperatures together:

$$
\mathrm{e}=\sum_{\mathrm{i}}^{\mathrm{n}} \sum_{\mathrm{j}}^{\mathrm{m}}\left[\mathrm{L}_{\mathrm{D}}{ }^{2}\left(\mathrm{t}_{\mathrm{j}}, \mathrm{T}_{\mathrm{i}}\right)-4 * \mathrm{t}_{\mathrm{j}} * \mathrm{D}_{\mathrm{o}} * \exp \left(-\mathrm{E}_{\mathrm{a}} /\left(\mathrm{K}^{*} \mathrm{~T}_{\mathrm{i}}\right)\right)\right]^{2}
$$

where $m$ is the number of the experimental datasets at a specific annealing temperature and $n$ is the number of different annealing temperatures.

The fitness function for the experimental datasets at a specific annealing temperature i.e. at individual annealing temperature is:

$$
\mathrm{e}=\sum_{\mathrm{j}}^{\mathrm{m}}\left[\mathrm{L}_{\mathrm{D}}^{2}\left(\mathrm{t}_{\mathrm{j}}, \mathrm{T}_{\mathrm{i}}\right)-4 * \mathrm{t}_{\mathrm{j}} * \mathrm{D}_{\mathrm{o}} * \exp \left(-\mathrm{E}_{\mathrm{a}} /\left(\mathrm{K}^{*} \mathrm{~T}_{\mathrm{i}}\right)\right)\right]^{2}
$$

where $m$ is the number of the experimental datasets at a specific annealing temperature. 


\section{Testing and Results}

The analysis of Photoluminescence measurement data for $10 \mathrm{~nm}$ single quantum well samples of $\operatorname{In}_{0.2} \mathrm{Ga}_{0.80}$ As in thick GaAs barriers annealed at temperatures $1173 \mathrm{~K}, 1223 \mathrm{~K}, 1273$ $\mathrm{K}$ and $1323 \mathrm{~K}$ shown in Figure 1 has been performed for estimating the activation energy, $\mathrm{E}_{\mathrm{a}}$, and the interdiffusion prefactor, $\mathrm{D}_{\mathrm{o}}$ by using the Real coded Quantum inspired Evolutionary Algorithm. The diffusion lengths squared versus the anneal time data in Figure 1 has been quoted from Gillin et al. [2].

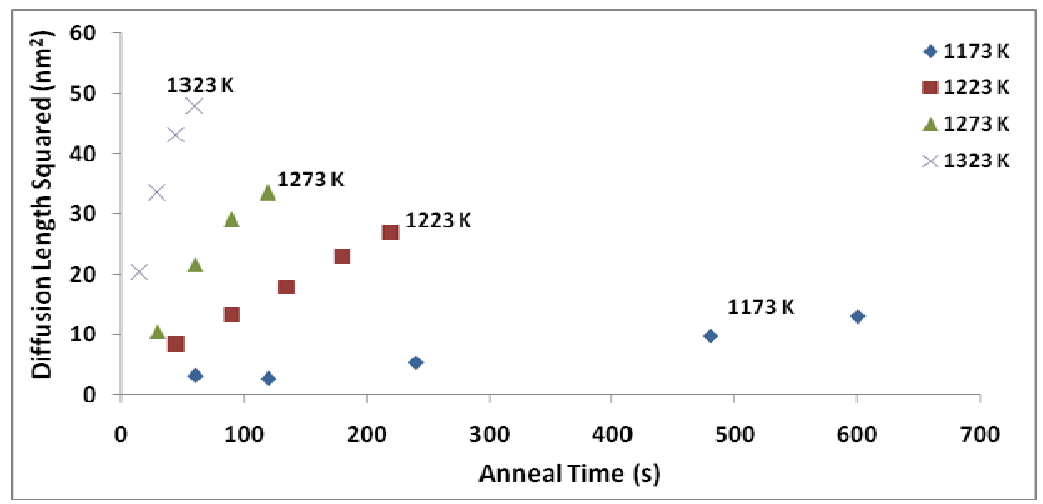

Figure 1: Photoluminescence measurement data set for 10nm single quantum well samples of $\mathrm{In}_{0.2} G a_{0.80} A s / G a A s$ annealed at $1173 \mathrm{~K}, 1223 \mathrm{~K}, 1273 \mathrm{~K}$ and $1323 \mathrm{~K}$ as diffusion length squared as a function of the annealing time [2].

One hundred independent runs have been performed in order to obtain $E_{a}$ and $D_{o}$ for each annealing temperature data individually (by using the equation (10)) as well as collectively (i.e. by taking all the data at all temperatures simultaneously using the equation (9)) by using ARQiEA.

ARQiEA was implemented in ' $\mathrm{C}$ ' programming language and run on an IBM Workstation with Pentium-IV 2.4 GHz processor, 2 GB RAM and Windows XP platform.

A total of five test-cases have been identified for testing purposes i.e. four individual PL measurement data sets at each annealing temperature and one collective data set [5]. The testing has been performed for determining the stability and efficiency of the proposed algorithm. The stability has been determined by statistically analyzing the quality of the solutions produced for each test-case in one hundred independent runs. The efficiency has been determined by the statistical analysis of the number of function evaluations performed in reaching the optimum in one hundred independent runs in each of the five test-cases. Further, convergence graphs have also been plotted for each of the test-cases.

Table 1 presents the statistical results for all the individual and collective test-cases for the estimation of the interdiffusion parameters, which indicates the stability of the algorithm. Best, Median, Worst, Mean and Standard Deviation (SD) of the Error in estimation of the diffusion length squared $\left(\mathrm{nm}^{2}\right)$ in one hundred independent runs of the proposed algorithm are also reported in Table I for all the test-cases. 
ARQiEA is a stable algorithm as it is able to reach the optima in all one hundred independent runs of all the five test-cases analyzed. The negligible value of the standard deviation is an indicator of the stability of the algorithm.

Table 2 presents the efficiency of ARQiEA by giving the statistical summary of the number of the function evaluations required for reaching the optima. The minimum number of the function evaluations is about one hundred fifty for the data set with $1173 \mathrm{~K}$ annealing temperature in the individual test case. The maximum number of function evaluations is about two thousand and two hundred for the data set with $1223 \mathrm{~K}$ annealing temperature in the individual test-case.

Table 1: Results of Ea and Do parameter estimation and the corresponding errors for all the five test-cases described in the text using ARQiEA

\begin{tabular}{|c|c|c|c|c|c|c|c|}
\hline Temperature & Ea & Do $x ~ 10^{-3}$ & \multicolumn{5}{|c|}{ Error $\left(\mathrm{nm}^{4}\right)$} \\
\cline { 4 - 8 }$(\mathrm{K})$ & $(\mathrm{eV})$ & $\left(\mathrm{cm}^{2} / \mathrm{s}\right)$ & Best & Median & Worst & Mean & SD \\
\hline 1173 & 3.090 & 1.00 & 4.426 & 4.426 & 4.426 & 4.426 & $6.24 \mathrm{E}-15$ \\
\hline 1223 & 3.031 & 0.99 & 12.073 & 12.073 & 12.073 & 12.073 & $5.36 \mathrm{E}-15$ \\
\hline 1273 & 3.061 & 1.00 & 24.759 & 24.759 & 24.759 & 24.759 & $2.50 \mathrm{E}-14$ \\
\hline 1323 & 3.057 & 1.00 & 133.872 & 133.872 & 133.872 & 133.872 & $2.57 \mathrm{E}-13$ \\
\hline Collective & 3.056 & 1.00 & 302.267 & 302.267 & 302.267 & 302.267 & $6.78 \mathrm{E}-12$ \\
\hline
\end{tabular}

Table 2: Results of statistical analysis of efficiency of ARQiEA in all the five test-cases described in the text

\begin{tabular}{|c|c|c|c|c|c|}
\hline \multirow{2}{*}{$\begin{array}{c}\text { Temperature } \\
(\mathrm{K})\end{array}$} & \multicolumn{5}{|c|}{ No. of Function Evaluations } \\
\cline { 2 - 6 } & Best & Median & Worst & Mean & SD \\
\hline 1173 & 153 & 758 & 1783 & 779.60 & 295.06 \\
\hline 1223 & 246 & 935 & 2204 & 951.04 & 307.40 \\
\hline 1273 & 227 & 706 & 1897 & 734.00 & 269.35 \\
\hline 1323 & 305 & 933 & 1464 & 901.35 & 226.16 \\
\hline Collective & 1061 & 4184 & 8694 & 4337.5 & 1992.81 \\
\hline
\end{tabular}

The minimum number of function evaluation is about one thousand and fifty for collective test-case. The maximum number of function evaluation is about eight thousand and seven hundred for the collective test-case. It is logical that collective test-case would require more number of function evaluations as there are almost four times the numbers of data elements in the collective test-case than in the individual test-cases.

Figure 2 - 6 show the convergence graphs for all the five test-cases for the median of one hundred independent runs. The graphs have been plotted with Error as a function of the number of function evaluations.

Figure 2 shows the convergence curve for the dataset with $1173 \mathrm{~K}$ annealing temperature, which indicates that ARQiEA reaches in the vicinity of the optimum i.e. within $1 \%$ of the 
optimum value in less than two hundred and fifty function evaluations. Figure 3 shows the convergence curve for the dataset with $1223 \mathrm{~K}$ annealing temperature, which indicates that ARQiEA reaches in the vicinity of the optimum i.e. within $1 \%$ of the optimum value in less than three hundred function evaluations. Figure 4 shows the convergence curve for the dataset with $1273 \mathrm{~K}$ annealing temperature, which indicates that ARQiEA reaches in the vicinity of the optimum i.e. within $1 \%$ of the optimum value in less than four hundred function evaluations. Figure 5 shows the convergence curve for the dataset with $1323 \mathrm{~K}$ annealing temperature, which indicates that ARQiEA reaches in the vicinity of the optimum i.e. within $1 \%$ of the optimum value in less than three hundred and fifty function evaluations.

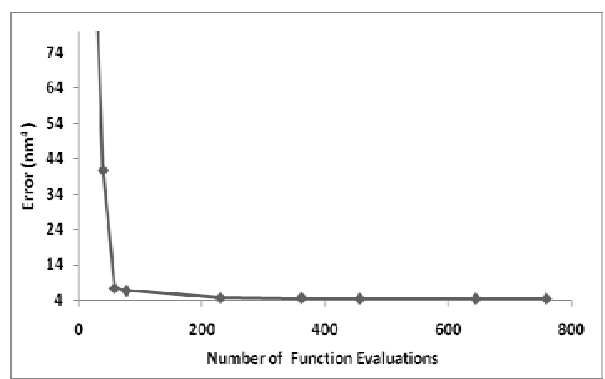

Figure 2: Convergence graph of median run for dataset with $1173 \mathrm{~K}$ annealing temp.

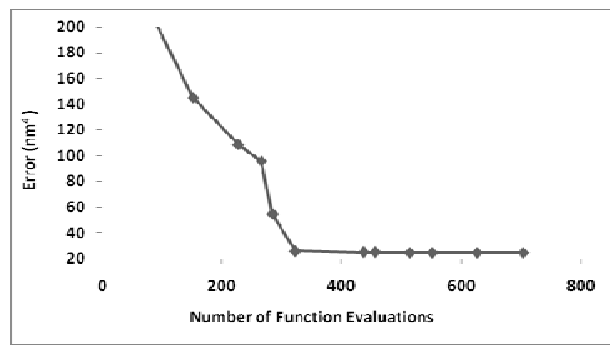

Figure 4: Convergence graph of median run for dataset with $1273 \mathrm{~K}$ annealing temp.

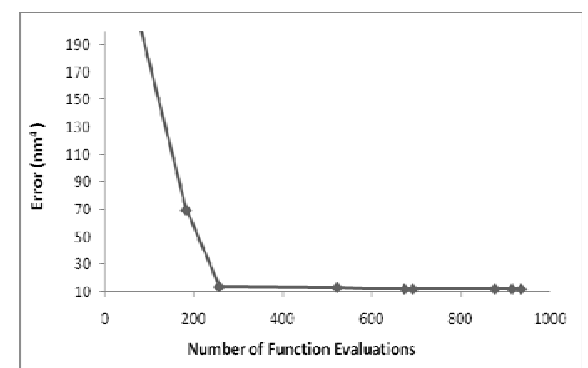

Figure 3: Convergence graph of median run for dataset with $1223 \mathrm{~K}$ annealing temp.

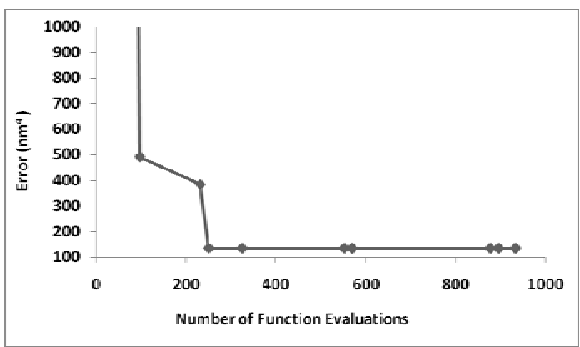

Figure 5: Convergence graph of median run for dataset with $1323 \mathrm{~K}$ annealing temp.

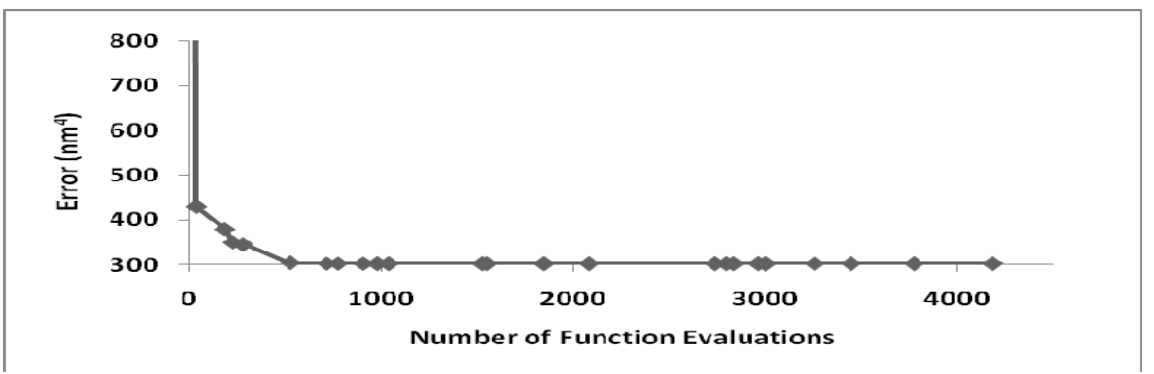

Figure 6: Convergence graph of median run for Collective Run

Figure 6 shows the convergence curve for collective run, which indicates that ARQiEA reaches in the vicinity of the optimum i.e. within $1 \%$ of the optimum value in less than one 
thousand function evaluations. Table 2 along with Figures $2-6$ show that ARQiEA is efficient and quickly reaches the vicinity of the optimum.

Table 3 presents comparison of ARQiEA with other techniques like Genetic Algorithm (GA) [5] and Least Square Analysis (LSA) method [2] on Error and interdiffusion coefficient $\mathrm{D}(\mathrm{T})$ given by equation (7) with respect to the best solution.

Table 3: Comparative Results of ARQiEA, GA [5] and LSA [2]

\begin{tabular}{|c|c|c|c|c|c|c|}
\hline \multirow[b]{2}{*}{$\begin{array}{c}\text { Temperature } \\
\text { (K) }\end{array}$} & \multicolumn{2}{|r|}{ ARQiEA } & \multicolumn{2}{|r|}{ GA [5] } & \multicolumn{2}{|r|}{ LSA [2] } \\
\hline & $\begin{array}{l}\text { Error } \\
\left(\mathrm{nm}^{4}\right)\end{array}$ & $\begin{array}{c}\mathrm{D}(\mathrm{T}) \times 10^{-16} \\
\left(\mathrm{~cm}^{2} / \mathrm{s}\right)\end{array}$ & $\begin{array}{l}\text { Error } \\
\left(\mathrm{nm}^{4}\right)\end{array}$ & $\begin{array}{c}\mathrm{D}(\mathrm{T}) \times 10^{-16} \\
\left(\mathrm{~cm}^{2} / \mathrm{s}\right)\end{array}$ & $\begin{array}{l}\text { Error } \\
\left(\mathrm{nm}^{4}\right)\end{array}$ & $\begin{array}{c}\mathrm{D}(\mathrm{T}) \times 10^{-16} \\
\left(\mathrm{~cm}^{2} / \mathrm{s}\right)\end{array}$ \\
\hline 1173 & 48.41 & 0.74 & 25.18 & 0.68 & 10.92 & 0.46 \\
\hline 1223 & 90.93 & 2.55 & 146.58 & 2.35 & 73.52 & 2.63 \\
\hline 1273 & 28.96 & 7.97 & 28.45 & 7.36 & 99.65 & 6.34 \\
\hline 1323 & 133.99 & 22.84 & 159.03 & 21.21 & 714.67 & 15.4 \\
\hline Collective & 302.28 & - & 359.26 & - & 898.77 & - \\
\hline
\end{tabular}

Table 3 shows that ARQiEA performs better than GA and LSA on overall error in collective test case. GA is better than LSA on the overall error in the collective test case. In case of individual data sets, the performance of ARQiEA is better than GA on $1223 \mathrm{~K}$ and $1323 \mathrm{~K}$ and LSA on 1273 and 1323.

\section{Conclusion and Future Work}

A new Adaptive real coded Quantum inspired Evolutionary Algorithm is used for analyses of Photoluminescence measurement data from interdiffused Quantum Wells. The algorithm uses two qubits representation instead of one and utilizes the quantum entanglement and superposition principles hitherto not tapped. It does not require mutation or local heuristic for improving the solution quality but still provides result with less overall error than the LSA and GA approaches. Further, this method determines interdiffusion parameters directly, without using Arrhenius plot. Thus, ARQiEA is suitable for parameter estimation problems.

Future work would involve more applications of ARQiEA for parameter estimation in analysis of experimental data in High Energy Physics and other areas.

\section{Acknowledgement}

We are extremely grateful to Most Revered Chairman, Advisory Committee on Education, Dayalbagh, for continued guidance and support in our every endeavor.

We are thankful to Prof. O. M. Khreis, Electronic Engineering Department, Yarmouk University, Idoon, Irbid, Jordan for providing the experimental PL measurement data used in this study. We also thank anonymous reviewers for giving insightful comments and suggestions. 


\section{References}

[1] C. Dion, P. Desjardins, N. Shtinkov, F. Schiettekatte, P. J. Poole, S. Raymond, Effects of grown-in defects on interdiffusion dynamics in InAs/InP(001) quantum dots subjected to rapid thermal annealing, JAP 103, 083526 (2008); doi:10.1063/1.2905317.

[2] W. P. Gillin, D. J. Dunstan, K. P. Homewood, L. K. Howard, B. J. Sealy, Interdiffusion in InGaAs/GaAs quantum well structures as a function of depth, JAP 73, 3782 (1993); doi:10.1063/1.352884.

[3] S.S. Rao, W.P. Gillin, K.P. Homewood, Interdiffusion of the group-III sublattice in In-Ga-As-P/InGa-As-P and In-Ga-As/In-Ga-As heterostructures, Phys. Rev. B 50, 8071 (1994).

[4] O. M. Khreis, K. P. Homewood, W. P. Gillin, K. E. Singer, Intermixing in GaAsSb/GaAs single quantum wells, JAP 84, 4017 (1998); doi:10.1063/1.368583.

[5] O. M. Khreis, I.S. Al-Kofahi, A genetic algorithm analysis of photoluminescence experimental data from interdiffused quantum wells, Superlattices and Microstructures 37, 3, 193 (2005).

[6] L. Teodorescu, Evolutionary Computation in High Energy Physics, arXiv:0804.0369.

[7] A. E. Eiben, and J. E. Smith, Introduction to Evolutionary Computing, Springer (2003).

[8] Z. Michalewicz, D. B. Fogel, How To Solve It: Modern Heuristics, Springer, (2004).

[9] A. E. Eiben, R. Hinterding, Z. Michalewicz, Parameter Control in Evolutionary Algorithms, IEEE Trans. Evo. Co. 3, 2, 124 (1999).

[10] K.H. Han, J.H. Kim, Quantum--Inspired Evolutionary Algorithm for a Class of Combinatorial Optimization, IEEE Trans. Evo. Co. 6, 6, 580, (2002).

[11] A. Mani, C. Patvardhan, An Adaptive Quantum Evolutionary Algorithm for Engineering Optimization Problems, IJCA 1, 22, 45, (2010).

[12] M.A. Nielsen, I.L. Chuang, Quantum Computation and Quantum Information. Cambridge University Press, Cambridge (2006).

[13] S. Yang, M. Wang, L. Jiao, A Novel Quantum Evolutionary Algorithm and its Application, Proc. of IEEE CEC-2004 1, 820, (2004).

[14] A. Narayanan, M. Moore, Quantum-inspired genetic algorithms, Proc. of IEEE CEC-1996, 61, (1996).

[15] Han, K.H. and Kim, J.H. 2002. Quantum-Inspired Evolutionary Algorithms with a New Termination Criterion, HE Gate and Two Phase Scheme, IEEE Trans. Evo. Co. 8, 2, 156, (2004).

[16] M. D. Platelt, S. Schliebs and N. Kasabov, A Verstaile Quantum-inspired EvolutionaryAlgorithm, Proc. IEEE CEC 2007, 423, (2007).

[17] F. S. Alfares, I. I. Esat, Real-coded Quantum Inspired Evolutionary Algorithm Applied to Engg. Optimiz. Problems, Proc. $2^{\text {nd }}$ Int. Symp. On LAFMVV, 169, (2007).

[18] F. Herrera, M. Lozano, A. M. Sanchez, A Taxonomy for the crossover operator for Real-coded Genetic Algorithms, Int. J. Intelligent Systems 18, 309, (2003). 